CASE REPORT

\title{
Multifocal angiomyolipoma affecting the liver and lung without tuberous sclerosis
}

\author{
M Saito, T Tsukamoto, T Takahashi, K Sai, H Fujii, K Nagashima
}

J Clin Pathol 2004;57:221-224. doi: 10.1136/زcp.2003.10330

The occurrence of angiomyolipoma (AML) in tissue other than the kidney is uncommon, as is multiple AML developing exclusively in organs other than the kidney. This report describes a case in which AML occurred multifocally in the liver and lung, but spared the kidney, and which might have been associated with tuberous sclerosis complex (TSC). A Japanese woman underwent a partial hepatectomy for a suspected malignant liver tumour at the age of 57 . The tumour consisted predominantly of a trabecular arrangement of myoid cells with a sinusoidal pattern and inflammatory cell infiltration, and was diagnosed as a primary liver AML by HMB-45 immunoreactivity. Five years later, multiple nodules were found in both lungs, for which video assisted thoracic surgery was performed. The tumour showed a mixture of epithelioid cells containing HMB-45 positive material and mature lipocytes, and was subsequently diagnosed as AML. Molecular analysis of both lesions showed no allelic loss of the TSC1 and TSC2 regions. Molecular analysis of the tumours ruled out an association with TSC, and both liver and lung lesions displayed benign histological features, so that these were probably multifocal lesions of AML without TSC.

A ngiomyolipoma (AML) occurs most frequently in the kidney, where it is closely related to tuberous sclerosis complex (TSC). ${ }^{12}$ Occasionally, AML also occurs in other organs, most commonly the liver, but occurrence at other sites is extremely rare. ${ }^{3}$ Irrespective of the type of organ involved, the diagnosis can be established by the presence of a marker of melanogenesis, such as human melanoma black 45 (HMB-45) positive smooth muscle cells. ${ }^{1-4}$ Among the three AML cellular components of fat, smooth muscle, and vessels, the myomatous or myoid tumour cells are the most variable, showing spindle, intermediate, epithelioid, and pleomorphic morphology. ${ }^{5}$

Hepatic AML is relatively rare, but to date about 200 cases have been reported in the literature since the first report in 1976 by Ishak. $^{6-9}$ Solitary hepatic AML is not usually associated with TSC, in contrast to multiple or combined AMLs of the kidney and liver. ${ }^{3}$

\footnotetext{
"The occurrence of angiomyolipoma in the liver and the lung without concurrent incidence in the kidney is extremely rare, and only two such cases have been reported"
}

AML, lymphangioleiomyomatosis (LAM), and clear cell "sugar" tumours of the lung are thought to comprise a class of tumour derived from the perivascular epithelioid cell family. ${ }^{10}$ They are related to each other by their coexpression of HMB-45, ${ }^{11}$ and should be added to the spectrum of pulmonary manifestations of TSC. ${ }^{12}{ }^{13}$ However, the histogenetic differentiation of these tumours remains controversial.

Typical AML is benign, but malignant renal or hepatic AMLs have been described in exceptional cases. ${ }^{8} 1415$ However, the multiple, unilateral, and bilateral renal AMLs and tumours located in regional lymph nodes are thought to be multifocal lesions, rather than metastases. ${ }^{1}$ The occurrence of AML in the liver and the lung without concurrent incidence in the kidney is extremely rare, and only two such cases have been reported. ${ }^{16}{ }^{17}$ Here, we report the third case of combined AMLs of the liver and lung, consisting of a solitary liver AML and multiple lung AMLs without TSC. Furthermore, there was no loss of heterozygosity ( $\mathrm{LOH})$ of microsatellite markers at TSC1 (9q34) and TSC2 (16p13.3) in the liver and lung lesions, which suggested that there was no genetic association with TSC.

\section{CLINICAL SUMMARY}

A 57 year old Japanese woman was hospitalised in April 1995 with general fatigue and left hypochondrial back pain. The patient did not have a noteworthy family history of disease. An abdominal computerised tomography (CT) scan disclosed a solitary tumour approximately $6 \mathrm{~cm}$ in diameter located in the lateral segment of the liver. The tumour was visualised intensely in the arterial phase, but not in the portal venous phase, and therefore resembled a hepatocellular carcinoma. A simple cyst $1 \mathrm{~cm}$ in diameter was noted in the right kidney. Liver function tests and tumour markers were unremarkable. Tests for hepatitis B surface antigen and hepatitis C virus antibody were negative. Although malignant lymphoma was suspected, no diagnosis was established when an echo guided needle biopsy of the liver tumour was performed. A partial hepatectomy was performed in May 1995, and the histopathological diagnosis of the liver tumour was primary hepatic AML (fig 1A).

On subsequent chest CT scan, first performed in June 2000 (at age 62), five years after the resection of liver AML, multiple small nodules (maximum $10 \mathrm{~mm}$ in diameter, but mostly 2-5 $\mathrm{mm}$ ) were found widely distributed in bilateral lung areas (fig 1B). Video assisted thoracic surgery (VATS) was performed on one nodule in the right lung (segment 10) adjacent to the pleura to make a definitive diagnosis. The histological diagnosis of this lung nodule was also AML.

In March 2003, about eight years after surgery, AML had not recurred in the remaining liver, and new lesions had not developed in the kidney. The residual lung tumours had remained stable for about three years since VATS was performed. In addition, TSC signs, such as cutaneous lesions,

Abbreviations: $\mathrm{AML}$, angiomyolipoma; $\mathrm{CT}$, computerised tomography; HMB-45, human melanoma black 45; LAM, lymphangioleiomyomatosis; $\mathrm{LOH}$, loss of heterozygosity; PCR, polymerase chain reaction; SSCP, single strand conformation polymorphism; TSC, tuberous sclerosis; VATS, video assisted thoracic surgery 

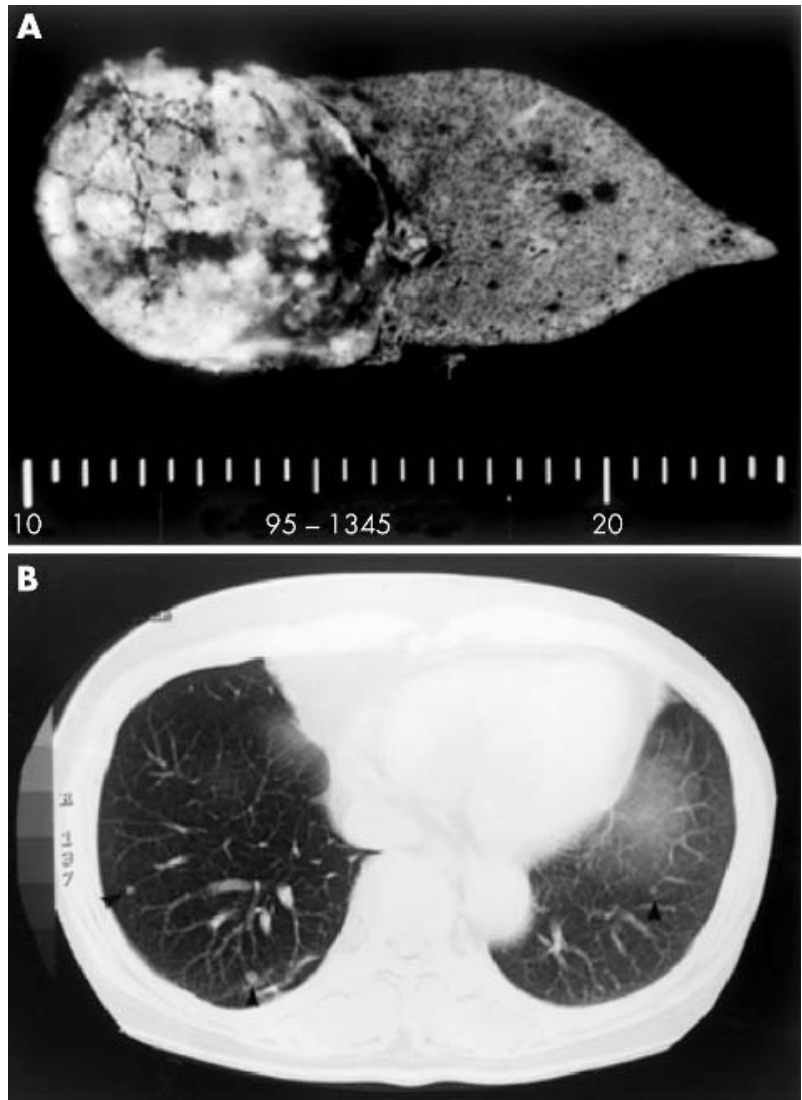

Figure 1 (A) Gross pathology of the liver tumour (May 1995). The cut surface of the tumour was well circumscribed and light brown in colour. (B) Chest computerised tomography scan (June 2000). Multiple small nodules about $5 \mathrm{~mm}$ in diameter were seen in the lung.

seizures, or mental retardation, were not observed, and the patient has remained symptom free at subsequent six monthly follow up examinations.

\section{MATERIALS AND METHODS}

The surgically resected material from the liver and lung was routinely stained with haematoxylin and eosin. Immunohistochemical staining was performed using the avidin-biotin-peroxidase technique, and the primary antibodies used were against HMB-45 (Dako, Glostrup, Denmark), $\alpha$ smooth muscle actin (Dako), actin HHF-35 (Dako), vimentin (Nichirei, Tokyo, Japan), S-100 (polyclonal; Dako), CD34 (Nichirei), Ki-67 (MIB-1; Immunotech, Marseille, France), and p53 (DO-7; Dako). Analysis of the immunohistochemical results showed the total percentage of myoid tumour cells staining positively.

After informed consent was obtained, $\mathrm{LOH}$ analysis of microsatellite markers at TSC1 (9q34) and TSC2 (16p13.3) was performed as described previously. ${ }^{18}$ Briefly, AML cells and normal liver parenchymal cells were separately microdissected from liver and lung lesions, and the extracted DNA was amplified by the polymerase chain reaction (PCR). The following microsatellite markers were selected to cover the chromosomal regions for the TSC1 and TSC2 $\operatorname{loci}^{19}$ : TSC1 at 9q34, and TSC2 at 16p13.3.

For simply detecting mutations of the p53 gene, exons 5-8 (mutation hotspots) from each DNA sample (with the nontumour background liver tissues as negative control) were amplified by PCR, followed by single strand conformation polymorphism (PCR-SSCP) analysis. ${ }^{20}$

\section{PATHOLOGICAL FINDINGS}

Macroscopically, the partial hepatectomy specimen contained a solitary, circumscribed, non-encapsulated mass surrounded by normal liver parenchyma. The tumour measured $60 \times 50 \times 30 \mathrm{~mm}$, and the cut surface was a uniform, light brown colour, with associated focal haemorrhages (fig 1).

On microscopic examination, the liver tumour consisted predominantly of solid sheets of myoid cells, which showed epithelioid, short spindle (intermediate), and spindle morphology. Large epithelioid cells contained granular material and often multiple perinuclear vacuoles, leaving cytoplasmic thread-like traces that have been described as a "spiderweb" cell pattern. ${ }^{7}$ A few large epithelioid cells showed a ganglion cell-like morphology. The intermediate cells were also vacuolated, and arranged in trabeculae, separated by sinusoidal structures composed of clear or blood filled spaces (fig 2A). In addition, aggregates of lymphoid cells were frequently seen (fig 2B). Typical mature adipocytes were scarce, but fat globules were seen among the tumour cells (fig 2A, arrows) and in sinusoidal spaces, as if floating in the spaces (fig $2 \mathrm{~A}$, asterisk). Thick walled blood vessels were also present, but were not angiomatous in nature. The nuclei of the tumour cells were normochromatic without pleomorphism, and mitoses were not seen. Tumour cells invading the portal tracts and hepatic veins were absent.

Lung tissue obtained by VATS contained two small nodules, $5 \mathrm{~mm}$ and $1 \mathrm{~mm}$ in diameter, with similar histological features. The lung nodules consisted of predominantly myoid cells, a few adipocytes, and vessels. Distinct epithelioid tumour cells with lightly stained eosinophilic cytoplasm arranged in broad solid sheets were seen. Fat was seen as more mature adipocytes (fig 2C), or as fine droplets within the light epithelioid cell cytoplasm. Hyalinous, thick walled blood vessels were also seen. Spindle shaped tumour cells were not seen. The nuclei of the epithelioid cells had slightly coarse chromatin without pleomorphism, and no mitoses were present.

\section{Immunohistochemical findings}

Both liver and lung AMLs showed intense cytoplasmic granular staining for HMB-45 (fig 2D, E). There was immunopositivity for $\alpha$ smooth muscle actin, and the lung AML showed more extensive positivity than the liver AML. Actin HHF-35 and vimentin were only focally present in some regions. In the liver AML, scattered cells were positive for the S-100 protein in areas of lymphocytic infiltration, but such cells were not found in the lung lesion. CD68 positivity was seen mainly in the sinusoidal macrophages, and the cytoplasm of some tumour cells. Positive staining for CD34 was limited to the endothelium, and staining for neurone specific enolase and cytokeratin was negative. The cell proliferation marker, Ki-67 labelling (MIB-1) index of the liver AML was approximately $1 \%$, and that of the lung was $3 \%$. Staining for the p53 protein was only found in a few cells, both in the liver and the lung.

\section{LOH analysis and p53 mutation}

None of the microsatellite markers revealed $\mathrm{LOH}$ (fig 3). Thus, there was retention of the alleles at the TSCl (9q34) and TSC2 loci (16p13.3) in all of the microdissected liver and lung AML foci.

None of the microdissected DNA samples showed the presence of the p53 mutation (exons 5-8) (data not shown).

\section{DISCUSSION}

The myomatous type of hepatic AML is composed predominantly of myoid cells, devoid of fat, and is more common than renal AML. ${ }^{7}$ In addition, some myomatous types of hepatic AML may exhibit distinct types of growth, such as 

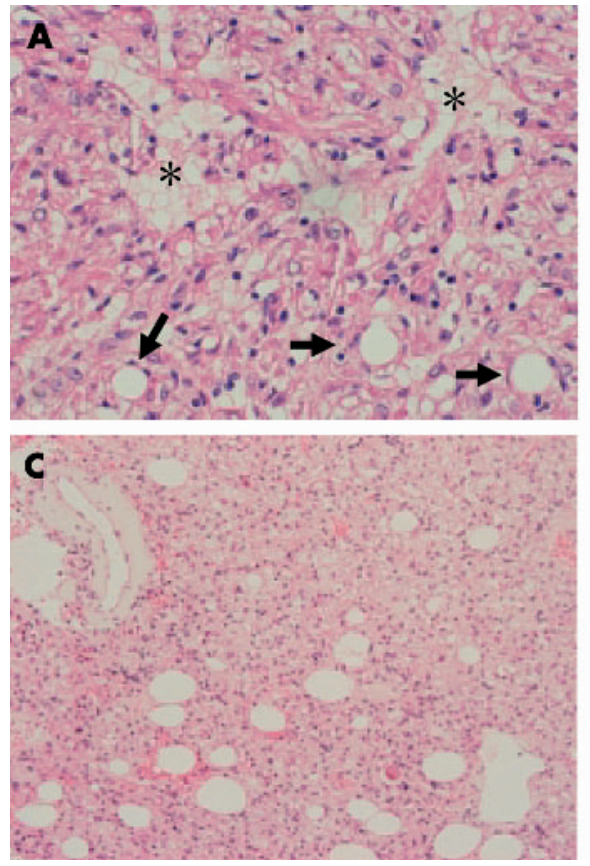
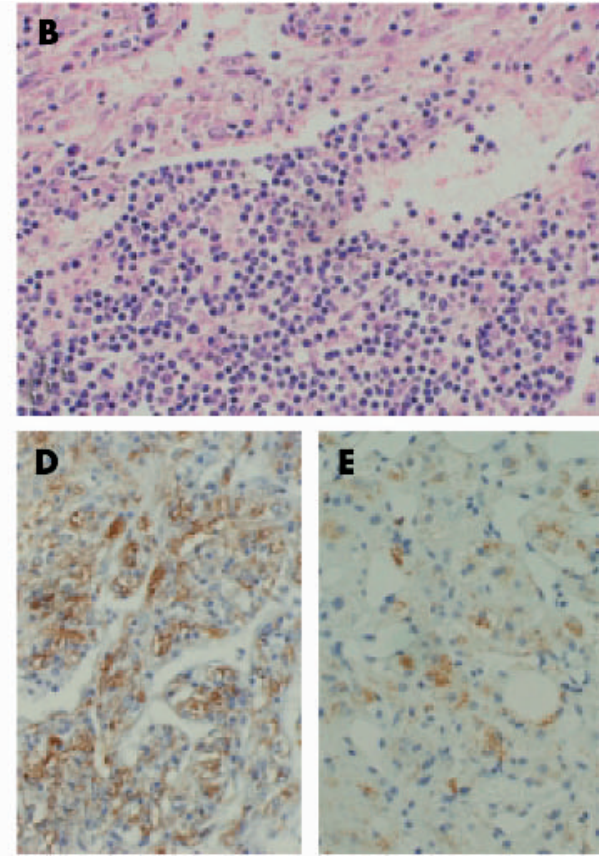

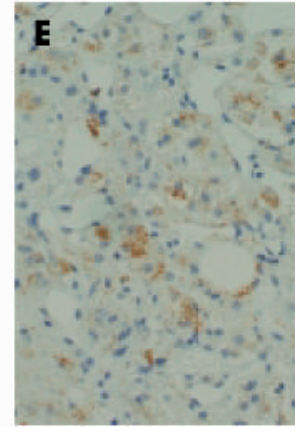

Figure 2 Histopathological and immunohistochemical appearance of the liver and lung angiomyolipoma (AML). (A) Short spindle or intermediate tumour cells were arranged in trabeculae separated by sinusoids. A few mature adipose cells were seen (arrows). In addition, fat globules were seen within sinusoids (asterisk). (B) Lymphocytic infiltration replaced myoid tumour cells, showing an inflammatory pattern. (C) Microscopic findings in the ung AML. Solid sheets of light, epithelioid tumour cells, hyalinised vessels, and mature adipocytes were seen. (D) Immunohistochemical staining of the hepatic tumour cells showing intense cytoplasmic granular staining for HMB-45. (E) Lung tumour cells contained HMB- 45 positive granular material in their cytoplasm. trabecular, ${ }^{4}$ pelioid, or inflammatory patterns. ${ }^{7}$ The liver tumour studied here was a myomatous type of hepatic AML with intermediate (short spindle) tumour cells showing trabecular and inflammatory patterns, and a scarcity of the typical fat component.

The occurrence of AML in the liver and the lung but sparing the kidney is unusual. In both our case and a recently reported case, ${ }^{1617}$ the lung AML cells had epithelioid morphology and expressed HMB-45. Recently, molecular analysis based on the concept of lyonisation has shown that AML exhibits clonal growth, despite morphological heterogeneity. ${ }^{21} 22$ Such an examination might detect clonality in our case, because in both the liver and the lung most of the tumour cells were myoid cells. The hepatic tumour in our case contained S-100 protein positive cells in addition to being HMB-45 positive. These S-100 positive cells might be dendritic cells because they were often seen in areas of lymphocytic infiltration.

"We suggest that our patient's tumours were multifocal angiomyolipoma affecting the liver and lung, and were not the result of a distant metastasis"

\section{Take home messages}

- This report describes the unique case of a 57 year old Japanese woman who had angiomyolipoma (AML) in the liver and the lung, with no signs of tuberous sclerosis complex or renal involvement

- Molecular analysis showed no allelic loss at the TSC1 and TSC2 regions

- The hepatic and lung AMLs were probably multifocal lesions, rather than pulmonary metastases originating in the liver

Clinically, the patient did not have TSC according to the criteria of Gomez, ${ }^{23}$ but recent studies indicate that sporadic LAM has LOH of TSC $2,{ }^{19}$ and can be a TSCl disease. ${ }^{18} \mathrm{LOH}$ of TSC1 and TSC2 was not detected in the liver and lung AML lesions, ruling out an association with TSC. Our findings suggest that lung AML is pathogenetically different to LAM, which is often associated with TSC. Further molecular analyses are needed to clarify this issue. According to a recent report, p53 mutation may play an important role in the
D16S283

D9S1198

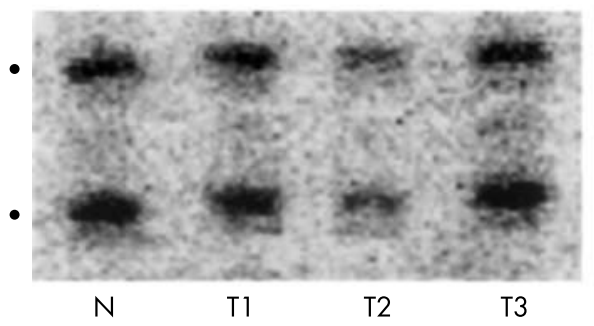

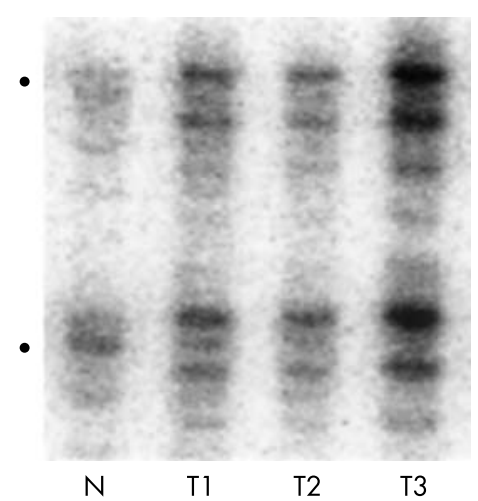

Figure 3 Representative microsatellite amplifications showing retention of 9q34 (D9S1 198) and 16p13.3 (D16S283). N, normal control DNA; T1, microdissected liver angiomyolipoma (AML) cells: T2, microdissected $5 \mathrm{~mm}$ lung $A M L$ nodule; T3, microdissected $1 \mathrm{~mm}$ lung AML nodule; dot, normal alleles. 
malignant transformation of renal AML. ${ }^{15}$ In our present case, using PCR-SSCP analysis, p53 mutation (exons 5-8) was not detected in the liver and lung lesions.

Although AML is essentially a benign tumour, occasional patients undergo a more unusual clinical course. Some cases suggest either an aggressive malignant AML, in which smooth muscle cells are seen together with cellular atypia, pleomorphic nuclei, and mitotic activity, or sarcomatous transformation, which exhibits vascular and lymphatic invasion. ${ }^{814} 15$ Based on the histological differences described above, coupled with the benign pathological findings and clinical course, we suggest that our patient's tumours were multifocal AML affecting the liver and lung, and were not the result of a distant metastasis.

In conclusion, we have described the unique case of a 57 year old Japanese woman with AML occurring in the liver and subsequently in the lung, with no signs of tuberous sclerosis complex or renal involvement. Molecular analysis showed no allelic loss at the TSC1 and TSC2 regions. We conclude that the liver and lung AMLs were multifocal lesions, rather than pulmonary metastases originating in the liver.

\section{ACKNOWLEDGEMENTS}

The authors thank Dr A Nonomura in the Pathology Section, Kanazawa University Hospital, Japan for expert commentary on our liver AML.

\section{Authors' affiliations}

M Saito, K Nagashima, Laboratory of Molecular and Cellular Pathology, Hokkaido University School of Medicine, Sapporo 0608638, Japan

T Tsukamoto, T Takahashi, Department of Pathology, Kushiro Rousai Hospital, Kushiro 085-8533, Japan

K Sai, Department of Internal Medicine, Kushiro Rousai Hospital

H Fujii, Department of Pathology, Juntendo University School of Medicine, Tokyo 113-8421, Japan

Correspondence to: $\mathrm{Dr} \mathrm{K}$ Nagashima, Laboratory of Molecular and Cellular Pathology, Hokkaido University School of Medicine, N-15, W-7, Kita-ku, Sapporo 060-8638, Japan; knagasi@med.hokudai.ac.jp

\section{REFERENCES}

1 Eble JN. Angiomyolipoma of kidney. Semin Diagn Pathol 1998;15:21-40.

2 Jimenez RE, Eble JN, Reuter VE, et al. Concurrent angiomyolipoma and renal cell neoplasia: a study of 36 cases. Mod Pathol $2001 ; 14: 157-63$.
3 Nonomura A, Mizukami Y, Takayanagi N, et al. Immunohistochemical study of hepatic angiomyolipoma. Pathol Int 1996;46:24-32.

4 Tsui WMS, Yuen AKT, Ma KF, et al. Hepatic angiomyolipoma with a deceptive trabecular pattern and HMB-45 reactivity. Histopathology 1992;21:569-73.

5 Nonomura A, Minato H, Kurumaya $\mathrm{H}$. Angiomyolipoma predominantly composed of smooth muscle cells: problems in histological diagnosis. Histopathology 1998;33:20-7.

6 Ishak KG. Mesenchymal tumors of the liver. In: Okuda K, Peters RL, eds. Hepatocellular carcinoma. New York: John Wiley, 1976:247-304.

7 Tsui WMS, Colombari R, Portmann BC, et al. Hepatic angiomyolipoma. A clinicopathologic study of 30 cases and delineation of unusual morphologic variants. Am J Surg Pathol 1999;23:34-48.

8 Dalle I, Sciot R, de Vos R, et al. Malignant angiomyolipoma of the liver: a hitherto unreported variant. Histopathology 2000;36:443-50.

9 Tang LH, Hui P, Garcia-Tsao G, et al. Multiple angiomyolipomata of the liver: a case report. Mod Pathol 2002;15:167-71.

10 Bonetti F, Pea M, Martignoni G, et al. Clear cell ("sugar") tumor of the lung is a lesion strictly related to angiomyolipoma-the concept of a family of lesions characterized by the presence of the perivascular epithelioid cells (PEC). Pathology 1994;26:230-6.

11 Fetsch PA, Fetsch JF, Marincola FM, et al. Comparison of melanoma antigen recognized by T-cell (MART-1) to HMB-45: additional evidence to support a common lineage for angiomyolipoma, lymphangiomyomatosis, and clear cell sugar tumor. Mod Pathol 1998;1 1:699-703.

12 Flieder DB, Travis WD. Clear cell "sugar" tumor of the lung: association with lymphangioleiomyomatosis and multifocal micronodular pneumocyte hyperplasia in a patient with tuberous sclerosis. Am J Surg Pathol 1997;21:1242-7.

13 Maruyama H, Seyama K, Sobajima J, et al. Multifocal micronodular pneumocyte hyperplasia and lymphangioleiomyomatosis in tuberous sclerosis with a TSC2 gene. Mod Pathol 2001;14:609-14.

14 Martignomi G, Pea M, Rigaud G, et al. Renal angiomyolipoma with epithelioid sarcomatous transformation and metastases. Demonstration of the same genetic defects in the primary and metastatic lesions. Am J Surg Pathol 2000;24:889-94.

15 Kawaguchi K, Oda Y, Nakanishi K, et al. Malignant transformation of renal angiomyolipoma. A case report. Am J Surg Pathol 2002;26:523-9.

16 Garcia TR, Mestre de Juan MJ. Angiomyolipoma of the liver and lung. A case explained by the presence of perivascular epithelioid cells. Pathol Res Pract 2002;198:363-7.

17 Kim NR, Chung MO, Park CK, et al. Pulmonary lymphangiomyomatosis and multiple hepatic angiomyolipomas in a man. Pathol Int 2003;53:231-5.

18 Sato T, Seyama K, Fujii H, et al. Mutation analysis of the TSC1 and TSC2 genes in Japanese patients with pulmonary lymphangioleiomyomatosis. J Hum Genet 2002;47:20-8.

19 Smolarek TA, Wessner LL, McCormack FX, et al. Evidence that lymphangiomyomatosis is caused by TSC2 mutations: chromosome 16p13 loss of heterozygosity in angiomyolipomas and lymph nodes from women with lymphangiomyomatosis. Am J Hum Genet 1998;62:810-15.

20 Oda T, Tsuda H, Sakamoto $M$, et al. Different mutations of the p53 gene in nodule-in-nodule hepatocellular carcinoma as a evidence for multistage progression. Cancer Lett 1994;83:197-200.

21 Saxena A, Alport EC, Custead S, et al. Molecular analysis of clonarity of sporadic angiomyolipoma. J Pathol 1999;189:79-84.

22 Flemming $\mathbf{P}$, Lehmann U, Becker T, et al. Common and epithelioid variants of hepatic angiomyolipoma exhibit clonal growth and share a distinctive immunophenotype. Hepatology 2000;32:213-17.

23 Gomez MR. Definition and criteria for diagnosis. In: Gomez MR, ed. Tuberous sclerosis complex, 3rd ed. New York: Oxford University Press, 1999:10-23. 